\title{
Altered functional connectivity is related to impaired cognition in left unilateral asymptomatic carotid artery stenosis patients
}

Shihao $\mathrm{He}^{1 \dagger}$, Ran Duan ${ }^{2 \dagger}$, Ziqi Liu' ${ }^{1}$, Cai Zhang ${ }^{3}$, Tian $\mathrm{Li}^{3}$, Yanchang Wei ${ }^{1}$, Ning Ma ${ }^{1}$ and Rong Wang ${ }^{1,4,2^{*}}$

\begin{abstract}
Background: Asymptomatic carotid artery stenosis (aCAS) impairs haemodynamic and cognitive functions; however, the relationship between these changes and brain network connectivity remains largely unknown. This study aimed to determine the relationship between functional connectivity and neurocognition in patients with aCAS.
\end{abstract}

Methods: We compared functional status in 14 patients with aCAS and 15 healthy controls using resting state functional magnetic resonance imaging sequences. The subjects underwent a full range of neuropsychological tests and a graphical theoretical analysis of their brain networks.

Results: Compared with controls, patients with aCAS showed significant decline in neuropsychological functions, particularly short-term memory (word-memory, $p=.046$ and picture-memory, $p=.014$ ). Brain network connectivity was lower in patients with aCAS than in the controls, and the decline of functional connectivity in aCAS patients was mainly concentrated in the left and right inferior frontal gyri, temporal lobe, left cingulate gyrus, and hippocampus. Decreased connectivity between various brain regions was significantly correlated with impaired short-term memory. Patients with aCAS showed cognitive impairment independent of known vascular risk factors for vascular cognitive impairment. The cognitive defects were mainly manifested in the short-term memory of words and pictures.

Conclusions: This study is the first of its kind to identify an association between disruption of functional connections in left carotid stenosis and impairment of short-term memory. The findings suggest that alterations in network connectivity may be an essential mechanism underlying cognitive decline in aCAS patients.

Clinical trial registration-URL: Unique identifier: 04/06/2019, ChiCTR1900023610.

\footnotetext{
* Correspondence: ronger090614@ccmu.edu.cn

'Shihao He and Ran Duan contributed equally to this work.

'Department of Neurosurgery, Beijing Tiantan Hospital, Capital Medical

University, Beijing 10070, China

${ }^{4}$ Center of Stroke, Beijing Institute for Brain Disorders, Beijing 100069, China

Full list of author information is available at the end of the article
}

(c) The Author(s). 2021 Open Access This article is licensed under a Creative Commons Attribution 4.0 International License, which permits use, sharing, adaptation, distribution and reproduction in any medium or format, as long as you give appropriate credit to the original author(s) and the source, provide a link to the Creative Commons licence, and indicate if changes were made. The images or other third party material in this article are included in the article's Creative Commons licence, unless indicated otherwise in a credit line to the material. If material is not included in the article's Creative Commons licence and your intended use is not permitted by statutory regulation or exceeds the permitted use, you will need to obtain permission directly from the copyright holder. To view a copy of this licence, visit http://creativecommons.org/licenses/by/4.0/ The Creative Commons Public Domain Dedication waiver (http://creativecommons.org/publicdomain/zero/1.0/) applies to the data made available in this article, unless otherwise stated in a credit line to the data. 


\section{Background}

Asymptomatic carotid artery stenosis (aCAS) is characterized by extracranial internal carotid artery stenosis in the ipsilateral carotid region, usually without a history of stroke or transient ischemic attack (TIA) [1]. Increasing evidence suggests that cognitive impairment may occur in not just symptomatic patients with a history of cerebral infarction or other cerebrovascular events, but also in those with aCAS [2]. Further, it has been shown that severe internal carotid artery stenosis $(>50 \%)$ is associated with a higher incidence of silent cerebral infarction [3]. In the mouse model of chronic cerebral insufficiency, although the mice did not develop motor dysfunction, their spatial learning ability through the maze test was reduced and they developed varying degrees of cognitive impairment [4]. These findings suggest that aCAS may not, in fact, be asymptomatic.

At present, functional neuroimaging is often used to reveal brain activity, which has become an important tool in cognitive research [5]. Brain network graph analysis provides an intuitive and attractive framework for studying the functional connections of brain information transmission and its correlation with behaviors [6], which may be used to explain some of the cognitive deficits in other diseases [7, 8]. However, few studies have investigated whether changes in the brain network are associated with altered cognitive function in patients with severe aCAS. Although cerebral hypoperfusion or infarction may be the cause of severe aCAS, the pathophysiological mechanism of brain function and network connectivity changes is still unclear [9-12].

This study aimed to determine the association between altered brain network connectivity and cognitive impairment in patients with aCAS using a graph theoretical analysis. We compared brain activities and cognitive function between 14 patients with severe aCAS and 15 matched healthy controls using neuropsychological tests and resting-state functional magnetic resonance imaging (rs-fMRI) scans.

\section{Materials and methods \\ Participants}

The participants in this prospective study consisted of 14 patients with unilateral aCAS who attended our institution between December 2018 and July 2019. After patients' baseline data were collated and analysed, 15 healthy volunteers were recruited based on age, sex, and years of education.

\section{Inclusion and exclusion criteria}

Inclusion criteria were as follows: [1] age 53-76 years; [2] left carotid artery stenosis $\geq 70 \%$ and right carotid artery stenosis < 50\%; [3] right-hand dominance; [4] no history of stroke, TIA, dementia, and depression; [5] an education level of primary school or above; [6] The minimental state exam (MMSE) score was between 27 and 30;

The exclusion criteria were as follows: [1] posterior circulation stroke/stroke syndrome; [2] neuropsychiatric diseases (e.g., Alzheimer's disease or Parkinson's disease), severe systemic diseases, or a history of stroke; [3] contraindications of MRI scans (e.g., metal implants); [4] take medications that may affect cognitive function; (5) hunger or fatigue; or [6] Cannot complete the test task independently.

\section{Neuropsychological assessments and MRI data processing methods}

For the neurocognitive function test, we adopted the method of He et al. [13] Specific cognitive tests are detailed in the supplementary materials. The neuropsychologists, who had no knowledge of each patient's clinical data, used a computer workstation to test the participants. Neuropsychological examination and functional magnetic resonance examination are separated by less than 5 days. In the correlation analysis of the connections between brain regions, the network-based-statistical analysis was first used to conduct a statistical test on the edge linkage of the two groups. NBS analysis and correction were carried out for the connecting edges based on the level of the mass. For the first time, 0.001 was used to retain all the connecting edges with $P$ value less than 0.001, and NBS correction was carried out for the remaining connecting edges with $P$ value card 0.05 . Detailed MRI parameters and fMRI data processing procedures are shown in the online supplementary materials.

\section{Statistical analyses}

Neuropsychological data were analysed using the Statistical Package for Social Sciences (IBM SPSS version 20.0 for Windows), and $p<0.05$ was considered statistically significant. For cognitive analysis, the control group was matched with the patient group in terms of age, sex, education, and hand dominance. Analysis of variance was conducted to exclude the influence of sex and risk factors on cognition performances. A two-sample t-test was performed to analyse differences between the patients and controls. All values are presented as mean \pm standard deviation. All participants' cognitive scores were correlated with significant brain network attributes using Pearson correlation analysis. The results for the model with statistically significant differences were also adjusted for multiple comparisons using the false discovery rate (Benjamini-Hochberg procedure).

\section{Results}

In order to reduce the limitations of multiple factors on the study results, 14 patients with unilateral aCAS (average age, 63.6 years) were included in this study through 
Table 1 Basic characteristics of study participants

\begin{tabular}{|c|c|c|c|}
\hline Characteristics & Patients $(n=14)$ & Controls $(n=15)$ & $p$-Values \\
\hline Age (years) & $63.6(6.246)$ & $62.4(5.804)$ & .584 \\
\hline \multicolumn{4}{|l|}{ Sex } \\
\hline Male: Female & $9: 5$ & $10: 5$ & $>.99$ \\
\hline Education (years) & $9.86(2.742)$ & $9.00(2.535)$ & .389 \\
\hline \multicolumn{4}{|l|}{ Risk factors (\%) } \\
\hline Hypertension & $11(78.6)$ & $8(53.3)$ & .245 \\
\hline Diabetes mellitus & $6(42.9)$ & $5(33.3)$ & .710 \\
\hline Ischaemic heart disease & $3(21.4)$ & $2(13.3)$ & .651 \\
\hline Hypercholesterolemia & $5(35.7)$ & $4(26.7)$ & .700 \\
\hline Smoking & $6(42.9)$ & $6(40)$ & $>.99$ \\
\hline
\end{tabular}

strict inclusion criteria, and 15 healthy controls (average age, 62.4 years) were matched according to age, sex, years of education, and underlying diseases. No significant difference in sex, educational status, and other clinical factors were observed between the two groups (Table 1). The carotid ultrasound diagnosis of the included patients was severe left carotid stenosis marked 70-99\%. There were no patients with significant unilateral occlusion.

Values are shown as the number of cases (\%) unless otherwise indicated.

The patients showed significantly poor performances on the neuropsychological tests, especially the shortterm memory test for Chinese words $(p=.046)$, and the picture memory test $(p=.014)$. Raven's Standard Progressive Matrices $(p=.063)$, executive function $(p=$ $.058)$, and choice RT $(p=.053)$ were low in the patient group, but the differences were not significant (Table 2).

Values are shown as the number (percentage) of cases unless otherwise indicated. The mean values are presented with the standard deviation. SD, standard deviation.

In the graph theory analysis, there were no significant differences between the patients and the healthy controls for the node-based network attributes and global parameters after the false discovery rate (FDR) test (Table 3 ), including assortativity $(p=.057)$, hierarchy $(p=.080)$, network efficiency $(p=.596)$, small-worldness $(p=.372)$, or synchronisation $(p=.881)$. The small-world attribute had a significant correlation with short-term word memory in the aCAS group $(\mathrm{r}=-.634, p=.015)$. The functional connections between the inferior frontal gyrus of the right hemisphere and the left orbital inferior frontal gyrus, hippocampus, superior temporal gyrus, and middle temporal gyrus were significantly low in the aCAS patients compared with the controls (Fig. 1).

The aCAS patients showed a significantly lower edge connection than the healthy controls $(p=.0316)$. A reduction in the edge property of the triangular part of the right inferior frontal gyrus (AAL label No. 14) connected to the other 17 nodes was observed.

As shown in Fig. 2, functional connections between the right triangular inferior frontal gyrus and the left orbital inferior frontal gyrus, the left hippocampus, the right superior temporal gyrus and the left middle temporal gyrus (the AAL brain regions of 14-15, 14-37, 14-84, and 14-85) were all correlated with word shortterm memory score, with positive correlation in the patient group and negative correlation in the control group. In the correlation analysis, functional connections between the the right triangular inferior frontal gyrus and the left superior temporal gyrus (the AAL brain regions of 14-81) were positively correlated with word short-term memory in the patient group and the control group.

Table 2 Neuropsychological assessments

\begin{tabular}{llll}
\hline & Patients, $\mathbf{n}=\mathbf{1 4}$ & Controls, $\mathbf{n}=\mathbf{1 5}$ & \\
\hline Variables & Mean (SD) & Mean (SD) & p-values \\
CRT_RT & $1159.36(902.439)$ & $641.80(172.601)$ & .053 \\
CRT_ACC & $97.00(6.288)$ & $99.20(1.424)$ & .221 \\
SPM & $11.71(5.757)$ & $15.53(4.853)$ & .063 \\
ROT & $9.57(5.893)$ & $13.53((7.190)$ & .118 \\
WWM1 & $6.07(1.817)$ & $6.73(1.438)$ & .285 \\
WWM2 & $4.36(1.336)$ & $4.80(1.699)$ & .444 \\
SUB & $29.64(9.145)$ & $34.40(7.189)$ & .130 \\
COMSUB & $13.29(5.239)$ & $13.07(6.193)$ & .919 \\
WORDM & $47.57(16.195)$ & $58.80(12.667)$ & $.046^{*}$ \\
PICTM & $66.29(7.878)$ & $73.07(5.994)$ & $.014^{*}$ \\
EXCUT1 & $0.57(3.228)$ & $1.47(2.924)$ & .440 \\
EXCUT2 & $0.07(3.912)$ & $-2.73(3.731)$ & .058 \\
\hline Abbreviatons: & SDD &
\end{tabular}

Abbreviations: SD, standard deviation; CRT_RT/ACC, Choice reaction time reaction time/ accuracy; SPM, Raven's Standard Progressive Matrices; ROT, Mental rotation; VWM, verbal working memory, digit span, 1, Recite in order, 2, Recite in reverse order; SUB, Simple subtraction; COMSUB, Complex subtraction; WORDM, word-memory; PICTM, picture-memory; EXCUT, Executive function, 1, same direction, 2, Opposite direction. ${ }^{*} p<.05$ 
Table 3 Differences in global attributes between the two study groups

\begin{tabular}{lllll}
\hline & Patients, $\boldsymbol{n}=\mathbf{1 4}$ Mean (SD) & Controls, $\boldsymbol{n}=\mathbf{1 5}$ Mean (SD) & $\boldsymbol{t}$-value & 1.985 \\
\hline Assortativity & $3.4142 \pm 0.8961$ & $2.8113 \pm 0.7369$ & -1.819 & 0.057 \\
Hierarchy & $-0.1791 \pm 0.3812$ & $0.0408 \pm 0.2633$ & -0.537 \\
Network efficiency & $0.2008 \pm 0.0093$ & $0.2024 \pm 0.0069$ & -0.151 & 0.080 \\
Synchronization & $-0.6175 \pm 0.6950$ & $-0.5814 \pm 0.5907$ & -0.908 \\
Small-worldness & $0.3927 \pm 0.0626$ & $0.4141 \pm 0.0642$ & 0.881 \\
\hline
\end{tabular}

Additionally, in the correlation analysis of functional connections between the right triangular inferior frontal gyrus and the Superior left margin (the AAL brain regions of 14-63) were all correlated with picture shortterm memory score, with negative correlation in the patient group and positive correlation in the control group.

As shown in Fig. 3, compared with the control group, functional connections related to brain network information transmission were more sparse in the patient group.

\section{Discussion}

Using rs-fMRI, this prospective study comprehensively elucidated the alterations in brain functional connectivity in patients with aCAS, and determined the correlation between altered brain functional connectivity and cognitive impairment. Our results suggest that shortterm memory and other cognitive functions decline in aCAS patients prior to the onset of clinical symptoms, and that changes in brain network connectivity may be associated with cognitive impairment.

A previous study observed that patients with aCAS gave a significantly poor performance on the MMSE and the Montreal Cognitive Assessment [14]. However, in our previous studies, we only observed minor changes in orientation, recall ability of three things, and language ability in aCAS patients. Therefore, we conducted a more detailed and systematic test using computer workstations to determine the specific extent of cognitive impairment in aCAS patients who had normal MMSE scores.

Cognitive impairment seems to selectively affect mental ability in patients, and some studies have demonstrated that stroke affects cognitive functions $[10,12$, 15]. This study found a statistically significant decline in short-term word and picture memory ability in aCAS patients, but not in reasoning and judgment ability, executive function, and reaction speed. Notably, calculation ability and spatial imagination ability were well-

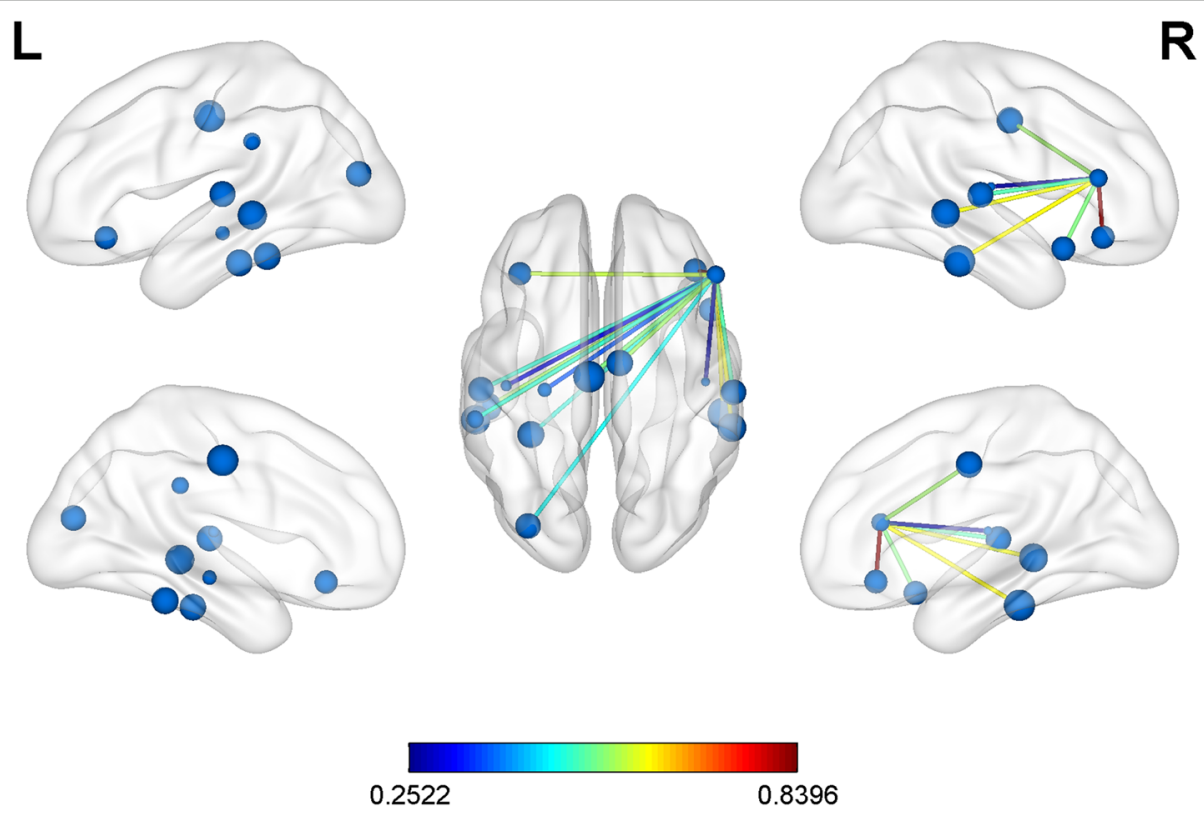

Fig. 1 Different connections between patients and healthy controls. The nodes and edges shown in the figure were significantly lower in patients than in healthy controls (aCAS < HC). The size of the node is the degree value; The range of colorbar represents the strength of a functional connection, from 0.25 to $0.83 \mathrm{aCAS}$, asymptomatic carotid artery stenosis; HC, healthy controls. 

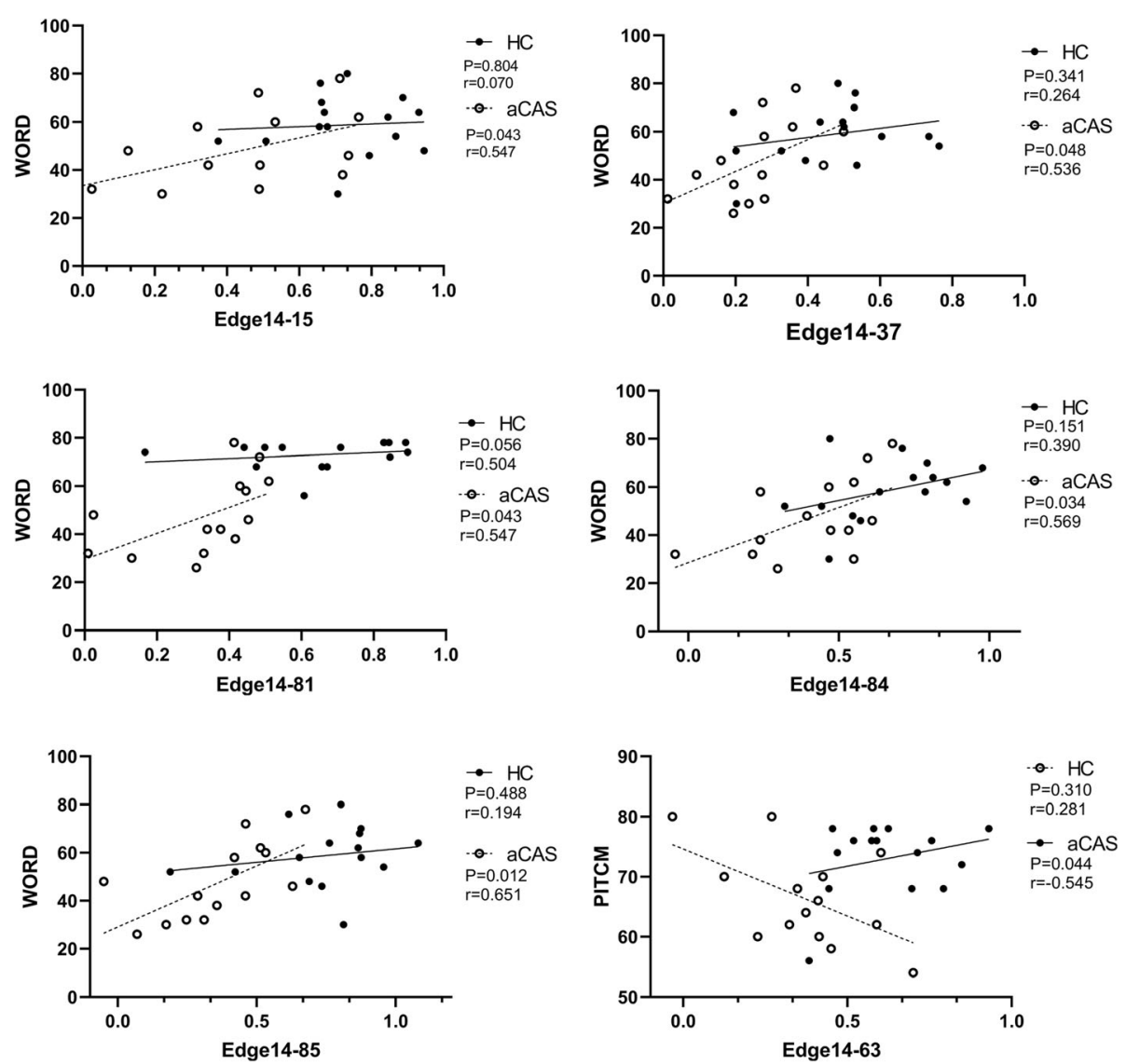

Fig. 2 Correlations between WORDM and PICM and connections. Six scatter charts for correlation analysis of edge attributes with short-term memory scores of words and pictures are shown. The regression lines were calculated in two groups. All probability values were adjusted by FDR correction. WORDM, word-memory; PICM, picture-memory; HC, healthy controls; aCAS, asymptomatic carotid artery stenosis; FDR, false discovery rate. Edge14: IFGtriang. R, Inferior frontal gyrus, triangular part; Edge15: ORBinf. L, Inferior frontal gyrus, orbital part; Edge37: HIP.L,Hippocampus; Edge81: STG. L, Superior temporal gyrus; Edge84: TPOsup. R, Temporal pole: superior temporal gyrus; Edge85: MTG. L, Middle temporal gyrus; Edge63: SMG. L, Supramarginal gyrus

preserved in these patients. Some pictures are easier to remember than words, and educational status may have some effects on the short-term memory of images and words. Additionally, studies have also demonstrated that decrease in image memory becomes less pronounced over time [16, 17]. Therefore, we further analysed the short-term memory decline after excluding the effects of age and education. We found that the decline in short-term memory in aCAS patients was still significant after adjusting for age and education. Therefore, the ability of short-term memory of words might be predominantly impaired in these asymptomatic patients. Numeric-span task was associated with short-term memory to some extent. Although the results of this task showed a decrease in the patient group, no statistically significant difference was found $(p=.285)$, which may be not related to the sample size of this study. The number - span task was often associated with executive function. The functional areas of short-term memory of numbers and words should be further studied.
One study found that the amplitude of low-frequency fluctuation values of the bilateral dorsomedial prefrontal cingulate cortex, praecuneus, superior temporal gyrus, and inferior parietal lobule decreased significantly in patients with aCAS, indicating a significant decrease in neural activity [18]. In this study, we used rs-fMRI data to construct the brain functional network to analyse the changes in network connectivity. Our study suggests that rs-fMRI may be used to detect an early decline in brain connections and predict the state of patients' memory.

A study found a markedly decreased blood oxygenation level-dependent correlation between opposite hemispheres when the ROI was predefined on the stenotic side (flipped to the left) in aCAS patients, suggesting a disruption of interhemispheric connectivity in these patients; [9] however, this finding is controversial because there are some differences between the left and right sides of the brain. Our graph theory analysis showed reduced connectivity between the brain 


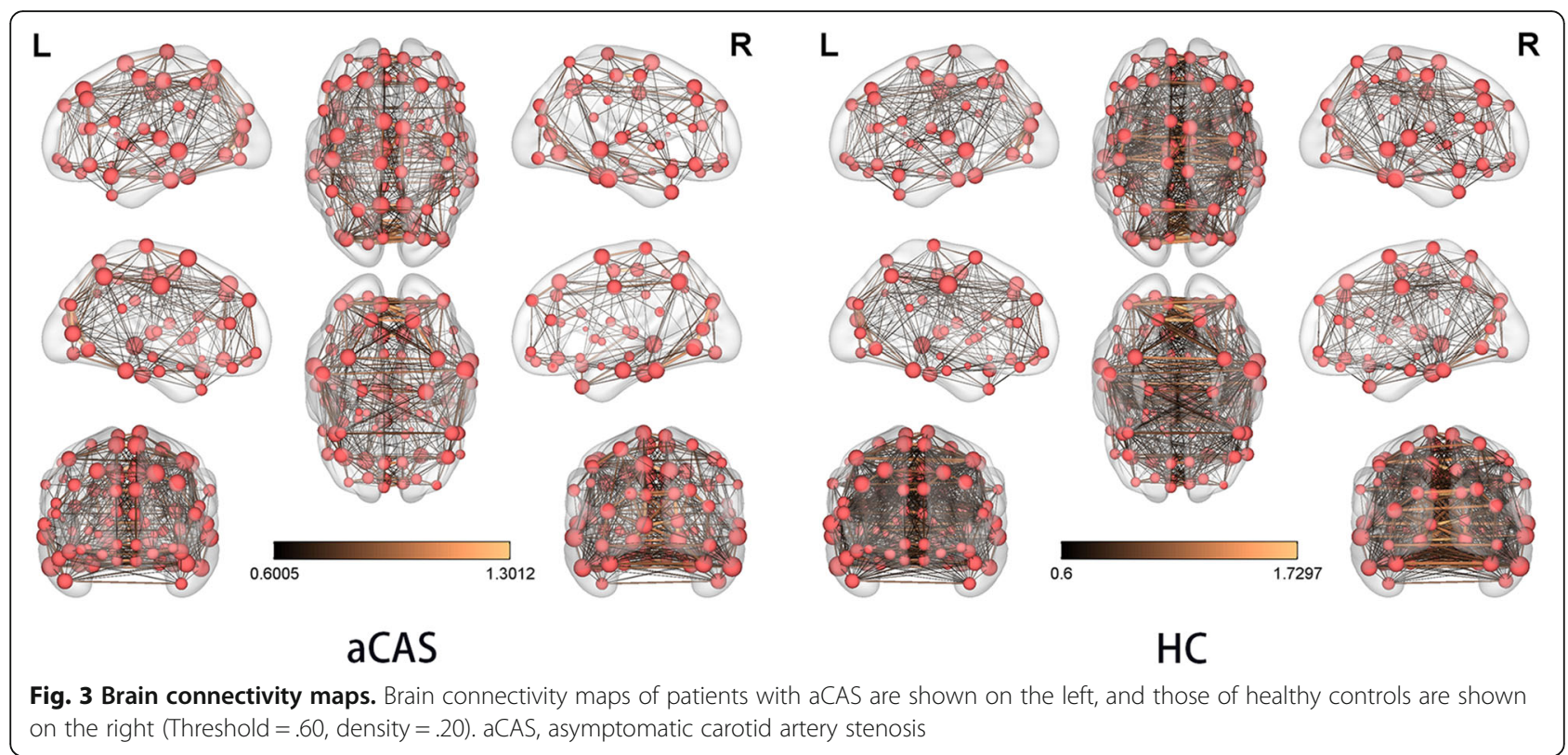

hemispheres in aCAS patients, and global efficiency was not significantly different between the aCAS patients and the healthy controls. These findings are similar to those observed in patients with symptomatic carotid artery stenosis, indicating that cerebral blood supply has a strong compensatory effect in chronic disease course [19]. It is thought that the brain of patients with aCAS could reconcile a good deal of short-range connections for segregation, while maintaining a sufficient number of remote connections to ensure processing integration. In our previous hemispheric network study, we found that the communication of CAS patients' coordination and information on the affected side of the brain was significantly impaired, but the contralateral brain network has shown the brain network compensation effect. In the left cerebral hemisphere of patients with CAO and CAS of the right carotid artery, connections between the Rolandic operculum and insula, median cingulate and paracingulate gyri and postcentral gyrus, and superior parietal gyrus and precuneus were stronger than those in normal controls [20]. Therefore, the results suggest that patients with carotid artery stenosis have different effects on the affected side, and that hemispheric template results may be more accurate for the global analysis of graph theory. In addition, the brain network connection will be reduced in the affected side and compensated in the healthy side.

There was no significant difference in node attributes of the graph theory analysis after FDR correction between the patient and control groups. However, the functional edge property was significantly lower in aCAS patients than in the controls $(p=.0316)$. Additionally, a reduced efficiency of functional connections in brain networks was observed in patients with aCAS (Fig. 3). In asymptomatic patients, the brain network is damaged and the node response is not significant, which may be slightly related to the illness, but also represents a higher sensitivity of functional connections.

The decline of functional connectivity in aCAS patients was mainly concentrated in the left and right inferior frontal gyri (IFG), temporal lobe, left cingulate gyrus, and hippocampus (Fig. 1). Our findings suggest that the weak association between these areas and the triangular part of the right inferior frontal gyrus may be directly related to short-term memory decline in patients. We further found that five functional connections between the triangular part of the inferior frontal gyrus (AAL no.14) and other brain regions were significantly correlated with short-term memory decline. Previous studies have suggested that IFG may be associated with affective empathy [21], and abnormalities in this functional connection have also been found in patients with depression [22]. At the same time, it has been pointed out that the two clusters in the posterior extra frontal flow (IFG) are functionally related to inhibition and implementation, while the two preclusters are related to reasoning and social cognition processes [23]. This area may be involved in different functions, and we found that reduced connections between this area and other brain areas may be associated with decreased short-term memory. This finding may suggest that cognitive function may be dominated not only by a single brain region or node, but also by multiple regions or networks.

The correlations between short-term word memory and the left orbital inferior frontal gyrus, left hippocampus, right superior temporal gyrus, left middle temporal 
gyrus, or right frontal lobe were reduced in aCAS patients, compared with healthy controls. At present, the function of the temporal lobe is not understood fully, but the sense of hearing is one of its main functions. It has been suggested that some visual resolution, as well as memory, language and some motor functions, may depend on the temporal lobe [24] [25]. Notably, increasing evidence suggests that the hippocampus may be associated with shortterm memory [26], and damage on both sides of the hippocampus may cause short-term memory loss, without affecting long-term memory. Therefore, the reduced brain network connections between the temporal lobe, hippocampus, and the triangular part of the right inferior frontal gyrus might be an important mechanism responsible for the short-term word-memory decline. These correlations were not significant in normal controls. It may also be related to insufficient samples in this study, and we will continue to conduct longitudinal studies.

Recent studies have shown that the left supramarginal gyrus is one of the key nodes of the short-term memory network involved in retaining an abstract representation of serial order information, independently from the content information [27]. The decline of functional connections between the right inferior frontal gyrus and the left supramarginal gyrus is related to the impairment of short-term memory $(\mathrm{r}=-.545, p=.044)$. This finding suggests that connections in these brain regions may indeed be important for short-term memory.

The purpose of this study was to increase the understanding of the mechanism of cognitive impairment by determining the correlation between cognitive impairment in aCAS and functional connectivity of brain networks, and to help define the criteria for intervention in the treatment of the disease. This study has some limitations. In order to reduce the limitation of multi-factors on the study results, we only included patients with unilateral left carotid artery stenosis in this study, and we intend to conduct a longitudinal study on patients with right carotid artery stenosis in the future. Additionally, the type of cognitive impairment may differ according to hand-dominance in the patients. Therefore, further studies including more patients with different handdominance and stenosis are needed to confirm our current findings.

\section{Conclusions}

We observed that the short-term memory of patients with left aCAS was highly correlated with an altered brain network connection. The alterations in network connections may be an important mechanism underlying the decline of cognitive function in patients with aCAS. Further, our findings indicate that rs-fMRI may be used to identify an early decline in brain connections and predict the state of patients' memory through correlation analysis of the brain network in various brain regions.

\section{Supplementary Information}

The online version contains supplementary material available at https://doi. org/10.1186/s12883-021-02385-4.

Additional file 1 SUPPLEMENTARY MATERIALS. The online supplementary materials include the introduction of the cognitive function test scale involved in this study as well as the detailed parameters of fMRI data, data pre-processing process, functional network analysis method and all the references involved.

\section{Acknowledgments}

My heartfelt thanks to my mentors for their unconditional trust and support. In addition, I am grateful to those who choose not to believe me in the difficult situation, because they have taught me to be stronger and work hard for my dream.

\section{Authors' contributions}

SH, RD, YC and ZQ acquired the MRI and clinical data. CZ, SH and ZQ performed the neuropsychological cognitive tests. SH, RD, ZQ,CZ,and TL analyzed and interpreted the data. SH,RD, ZQ, CZ, and YC drafted the manuscript. RW, SH, CZ and NM critically revised the manuscript for important intellectual content. SH, ZQ, CZ and TL performed the statistical analysis. RD, NM and RW supervised the study. All authors have read and approved the manuscript.

\section{Funding}

This study was supported by the Beijing municipal health system high-level health technical personnel training program (2015-3-041-DR) and Beijing Municipal Science \& Technology Commission (Z151100004015077-DR). The first funded tests of cognitive function and fMRI in patients and controls. The latter helped our researchers learn MRI analysis techniques.

\section{Availability of data and materials}

All data generated or analyzed during this study are included in the published article. Some or all data, models, or code generated or used during the study are available from the corresponding author by request.

\section{Declarations}

Ethics approval and consent to participate

The study is based on the principles expressed in the Declaration of Helsinki. Informed consent was signed by all participants. The institutional review Committee of Beijing Tiantan Hospital of Capital Medical University approved the study. (KYSQ2019-058-01)

Consent for publication

Not applicable.

\section{Competing interests}

The authors declare that the research was conducted in the absence of any commercial or financial relationships that could be construed as a potential conflict of interest.

\section{Author details}

${ }^{1}$ Department of Neurosurgery, Beijing Tiantan Hospital, Capital Medical University, Beijing 10070, China. ${ }^{2}$ Department of Neurosurgery, Peking University International Hospital, Beijing 102206, China. ${ }^{3}$ Collaborative Innovation Center of Assessment for Basic Education Quality, Beijing Normal University, Beijing 100875, China. ${ }^{4}$ Center of Stroke, Beijing Institute for Brain Disorders, Beijing 100069, China.

Received: 16 February 2021 Accepted: 1 September 2021

Published online: 13 September 2021

\section{References}

1. Prevention of disabling and fatal strokes by successful carotid endarterectomy in patients without recent neurological symptoms: randomised controlled trial. The Lancet. 2004;363(9420):1491-502.

2. Yoshizaki K, Adachi K, Kataoka S, Watanabe A, Tabira T, Takahashi K, et al. Chronic cerebral hypoperfusion induced by right unilateral common carotid 
artery occlusion causes delayed white matter lesions and cognitive impairment in adult mice. Exp Neurol. 2008;210(2):585-91. https://doi.org/1 0.1016/j.expneurol.2007.12.005.

3. Romero JR, Beiser A, Seshadri S, Benjamin EJ, Polak JF, Vasan RS, et al. Carotid artery atherosclerosis, MRI indices of brain ischemia, aging, and cognitive impairment. Stroke. 2009;40(5):1590-6. https://doi.org/10.1161/ STROKEAHA.108.535245.

4. Mansour A, Niizuma K, Rashad S, Sumiyoshi A, Ryoke R, Endo H, et al. A refined model of chronic cerebral hypoperfusion resulting in cognitive impairment and a low mortality rate in rats. J Neurosurg. 2018;131(3):892902. https://doi.org/10.3171/2018.3.JNS172274.

5. Stam CJ. Modern network science of neurological disorders. Nat Rev Neurosci. 2014;15(10):683-95. https://doi.org/10.1038/nrn3801.

6. Bassett DS, Zurn P, Gold J. On the nature and use of models in network neuroscience. Nat Rev Neurosci. 2018;19(9):566-78. https://doi.org/10.1038/ s41583-018-0038-8

7. Cheng W, Rolls ET, Gu H, Zhang J, Feng J. Autism: reduced connectivity between cortical areas involved in face expression, theory of mind, and the sense of self. Brain. 2015;138(Pt 5):1382-93. https://doi.org/10.1093/brain/a wV051.

8. Eijlers AJC, KAM, Thomas M. Wassenaar, et al. increased default-mode network centrality in cognitively impaired multiple sclerosis patients. Neurology. 2017:88:1-9.

9. Cheng H-L, Lin C-J, Soong B-W, Wang P-N, Chang F-C, Wu Y-T, et al Impairments in cognitive function and brain connectivity in severe asymptomatic carotid stenosis. Stroke. 2012;43(10):2567-73. https://doi.org/1 0.1161/STROKEAHA.111.645614.

10. Mellon L, Brewer L, Hall P, Horgan F, Williams D, Hickey A, et al. Cognitive impairment six months after ischaemic stroke: a profile from the ASPIRE-S study. BMC Neurol. 2015;15(1):31. https://doi.org/10.1186/s12883-015-0288-2.

11. Park J-Y, Kim Y-H, Chang WH, C-h P, Shin Y-I, Kim ST, et al. Significance of longitudinal changes in the default-mode network for cognitive recovery after stroke. Eur J Neurosci. 2014;40(4):2715-22. https://doi.org/10.1111/ ejn. 12640

12. Zhang P, Xu Q, Dai J, Wang J, Zhang N, Luo Y. Dysfunction of affective network in post ischemic stroke depression: a resting-state functional magnetic resonance imaging study. Biomed Res Int. 2014;2014:1-7. https:// doi.org/10.1155/2014/846830.

13. He S, Duan R, Liu Z, Ye X, Yuan L, Li T, et al. Characteristics of cognitive impairment in adult asymptomatic moyamoya disease. BMC Neurology. 2020;20(1)

14. Wang $T$, Xiao F, Wu G, Fang J, Sun Z, Feng H, et al. Impairments in brain perfusion, metabolites, functional connectivity, and cognition in severe asymptomatic carotid stenosis patients: an integrated MRI study. Neural Plast. 2017;2017:8738714-7. https://doi.org/10.1155/2017/8738714.

15. Bournonville C, Hénon H, Dondaine T, Delmaire C, Bombois S, Mendyk A-M, et al. Identification of a specific functional network altered in poststroke cognitive impairment. Neurology. 2018;90(21):e1879-e88. https://doi.org/1 0.1212 WNL.0000000000005553.

16. Morgan PL, Williams C, Ings FM, Hughes NC. Effects of valent image-based secondary tasks on verbal working memory. Q J Exp Psychol (Hove). 2018; 71(6):1440-56. https://doi.org/10.1080/17470218.2017.1329324.

17. Goetschalckx L, Moors P, Wagemans J. Image memorability across longer time intervals. Memory. 2018;26(5):581-8. https://doi.org/10.1080/09658211.2 017.1383435.

18. Xiao F, Wang T, Gao L, Fang J, Sun Z, Xu H, et al. Frequency-dependent changes of the resting BOLD signals predicts cognitive deficits in asymptomatic carotid artery stenosis. Front Neurosci. 2018;12. https://doi. org/10.3389/fnins.2018.00416.

19. Chang T-Y, Huang K-L, Ho M-Y, Ho P-S, Chang C-H, Liu C-H, et al. Graph theoretical analysis of functional networks and its relationship to cognitive decline in patients with carotid stenosis. J Cereb Blood Flow Metab. 2015; 36(4):808-18. https://doi.org/10.1177/0271678X15608390.

20. He S, Liu Z, Xu Z, Duan R, Yuan L, Xiao C, et al. Brain functional network in chronic asymptomatic carotid artery stenosis and occlusion: changes and compensation. Neural Plast. 2020;2020:9345602-11. https://doi.org/10.1155/2 020/9345602.

21. Shamay-Tsoory SG, Aharon-Peretz J, Perry D. Two systems for empathy: a double dissociation between emotional and cognitive empathy in inferior frontal gyrus versus ventromedial prefrontal lesions. Brain. 2009;132(Pt 3): 617-27. https://doi.org/10.1093/brain/awn279.
22. Rolls ET, Cheng W, Du J, Wei D, Qiu J, Dai D, et al. Functional connectivity of the right inferior frontal gyrus and orbitofrontal cortex in depression. Soc Cogn Affect Neurosci. 2020;15(1):75-86. https://doi.org/10.1093/scan/nsaa 014.

23. Hartwigsen G, Neef NE, Camilleri JA, Margulies DS, Eickhoff SB. Functional segregation of the right inferior frontal gyrus: evidence from Coactivationbased Parcellation. Cereb Cortex. 2019;29(4):1532-46. https://doi.org/10.1 093/cercor/bhy049.

24. Fernandez VG, Asarnow R, Narr KL, Subotnik KL, Kuppinger H, Fogelson D, et al. Temporal lobe thickness and verbal memory in first-degree relatives of individuals with schizophrenia. Schizophr Res. 2018;199:221-5. https://doi. org/10.1016/j.schres.2018.02.038.

25. Doty RL, Tourbier I, Neff JK, Silas J, Turetsky B, Moberg P, et al. Influences of temporal lobe epilepsy and temporal lobe resection on olfaction. J Neurol. 2018;265(7):1654-65. https://doi.org/10.1007/s00415-018-8891-y.

26. Tajerian M, Hung V, Nguyen H, Lee G, Joubert LM, Malkovskiy AV, et al. The hippocampal extracellular matrix regulates pain and memory after injury. Mol Psychiatry. 2018;23(12):2302-13. https://doi.org/10.1038/s41380-018-02 09-z.

27. Guidali G, Pisoni A, Bolognini N, Papagno C. Keeping order in the brain: the supramarginal gyrus and serial order in short-term memory. Cortex. 2019; 119:89-99. https://doi.org/10.1016/j.cortex.2019.04.009.

\section{Publisher's Note}

Springer Nature remains neutral with regard to jurisdictional claims in published maps and institutional affiliations.
Ready to submit your research? Choose BMC and benefit from:

- fast, convenient online submission

- thorough peer review by experienced researchers in your field

- rapid publication on acceptance

- support for research data, including large and complex data types

- gold Open Access which fosters wider collaboration and increased citations

- maximum visibility for your research: over $100 \mathrm{M}$ website views per year

At $\mathrm{BMC}$, research is always in progress.

Learn more biomedcentral.com/submissions 